\title{
Influence of water on the dielectric properties, electrical conductivity and microwave absorption properties of amorphous yellow dextrin
}

\author{
Adrian Radoń (D) Patryk Włodarczyk
}

Received: 12 August 2018/ Accepted: 12 February 2019/Published online: 20 February 2019

(C) The Author(s) 2019

\begin{abstract}
Electrical conductivity as well as dielectric and microwave absorption properties of yellow dextrin were described. The studied material was in the form of milled and pressed tablets. It was found that the minor addition of water $(5.4 \mathrm{wt} \%$ of water content) significantly changes the properties of tested absorber. The shift of $\gamma$-relaxation was observed for water-saturated amorphous yellow dextrin. It was also confirmed, that the activation energy of this process has been significantly increased by a slight addition of water $(0.45-0.62 \mathrm{eV})$. Mobility of water in the yellow
\end{abstract}

dextrin-water system is also related to the increased conductivity of the sample. The yellow dextrin was found to be a great microwave absorber. Addition of water causes also the increase of microwave absorption properties (decrease of reflection loss from -2.37 to $-17.29 \mathrm{~dB}$ and shielding efficiency from -9.09 to $-36.84 \mathrm{~dB}$ for $1 \mathrm{~cm}$ sample thickness). Moreover, the highest absorption frequency region is being shifted by water towards lower frequencies (from 4.78 to $2.83 \mathrm{GHz}$ ).

A. Radoń $(\bowtie) \cdot$ P. Włodarczyk Institute of Non-Ferrous Metals, Sowinskiego 5 St., 44-100 Gliwice, Poland e-mail: adrianr@imn.gliwice.pl 


\section{Graphical abstract}

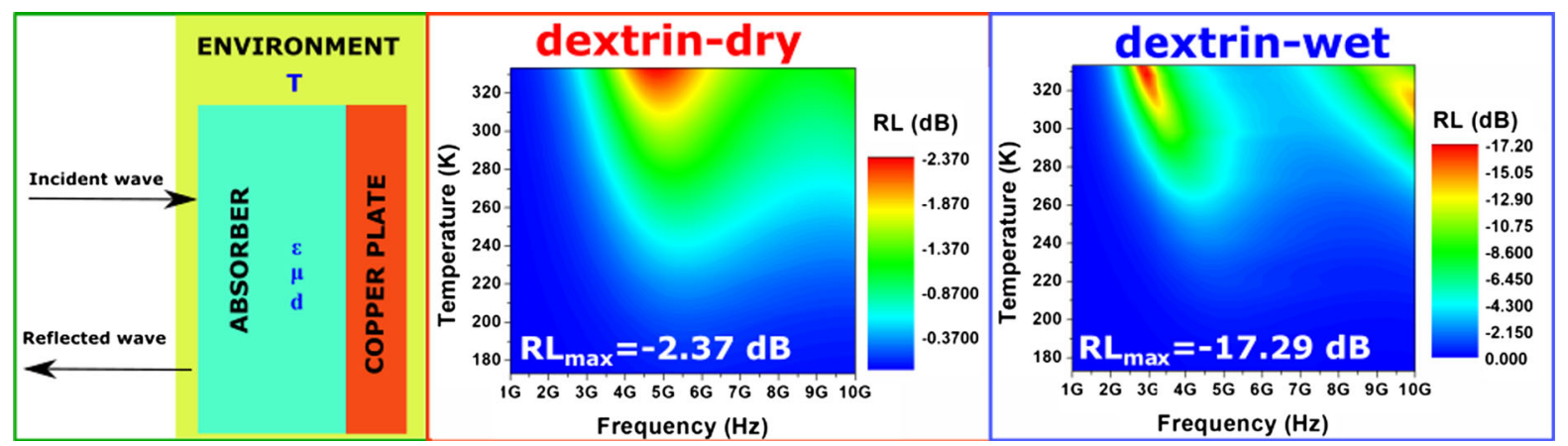

Keywords Dextrin - Microwave absorbers .

Relaxation · Electrical conductivity $\cdot$ EMI shielding properties

\section{Introduction}

Dextrins are mixtures of polymers of D-glucose units linked mainly by $\alpha-(1 \rightarrow 4)$ glycosidic bonds. They are a group of water-soluble carbohydrates, which are produced by the hydrolysis of glycogen or starch with chemical formula $\left(\mathrm{C}_{6} \mathrm{H}_{10} \mathrm{O}_{5}\right)_{n}$. Dextrins can be produced by a partial hydrolysis of starch using different methods such as acid hydrolysis, enzymatic hydrolysis or heat treatment. The most common pyrodextrins (dextrins produced by heating of dry starch) are white dextrin, yellow dextrin and British gums. The British gums are produced by using high temperature for a long period of time without or with addition of small amount of acid, whereas white dextrin can be produced by heating of starch at low temperature for a short period of time with the addition of acidic catalysts (Shrestha and Halley 2014). The yellow dextrin is formed when starch is heated at high temperature (423-493 K) by 6-18 $\mathrm{h}$ in the presence of acidic catalysts (Greenwood 1967). Under these conditions, transglycosylation and repolymerization of starch fragments occurs simultaneously (Terpstra et al. 2010). The characteristic yellow color can be related to the caramelization process occurring at that temperatures. Several kinds of dextrin, including yellow dextrin, white dextrin, maltodextrin, cyclodextrin and amylodextrin have found application due to their biocompatibility and solubility in water (Takatori et al. 2011; Silva et al. 2014). The yellow dextrin has found application as a binder in adhesive applications, extender in dyes and binder in ceramic industry, which is associated with high solubility in cold water. Until now many different works describe molecular dynamics of amorphous saccharides and their solutions (Weingärtner et al. 2001; Shinouda and Moteleb 2005; Kaminski et al. 2012). However, none of them are concentrated on electric conductivity and microwave absorption (MA) properties. Influence of water on the relaxation processes in saccharides was studied in great detail in different saccharides. Kaminski et al. have found that in saccharides that are similar to dextrin i.e. cyclodextrin, water decreases the activation barrier of the slow $\beta$-relaxation, while increasing the activation barrier of the $\gamma$-relaxation. This seems to be universal for saccharides. The $\beta$-relaxation is probably related to the rotation of monosaccharide units along the glycosidic linkage (Kaminski et al. 2008, 2012; Rachocki et al. 2005), while $\gamma$-relaxation can be ascribed to the reorientation of hydroxymethyl group $\left(-\mathrm{CH}_{2} \mathrm{OH}\right)$ (Kaminski et al. 2010). The water forms hydrogen bonds with hydroxyl groups of carbohydrate, suppressing internal hydrogen bonds in glycosidic bridge. Therefore, it allows for better mobility of glycosidic linkage and worse mobility of hydroxymethyl group. Change of hydrogen bond pattern can explain the behavior of relaxation processes in the sample saturated with water. Shinouda and Moteleb described also influence of water on different polysaccharides: hydrocellulose, hydroxypropyl cellulose and dextran (Shinouda and Moteleb 2005). They showed, that the presence of absorbed water significantly changes dielectric properties of polysaccharides. For example after evaporation of 
water, $\gamma$-relaxation appears and intensity of $\beta$-relaxation decreases in the dielectric spectrum. The similar results were obtained for different types of celluloses such as native cellulose and regenerated cellulose fibers (Einfeldt and Kwasniewski 2002). Moreover, the influence of moisture content on the dielectric properties of white and yellow dextrin were used by Boruch et al. (1988) to develop a method for determination of water content in dextrins. However, we were unable to find any work on the influence of water on electrical conductivity and electrode polarization process at low frequencies.

The organic compounds with polar groups such as saccharides can be used as microwave absorbers. The energy of electromagnetic wave can be transferred to the degrees of freedom related with the $\gamma$ or $\beta$ relaxation. Wlodarczyk et al. (2015) indicated that sucrose can be a good microwave absorber due to the fact that it has $\gamma$-relaxation characterized by high dielectric strength. As this process is sensitive to both water content and temperature, these two effects need to be taken into account in the studies of shielding properties. Moreover, low-dense polysaccharides can be successfully used as light electromagnetic interference (EMI) shielding materials. Until now, many different EMI shielding materials with high microwave absorption properties were developed and tested. The EMI shielding properties of conductive materials (metals, intrinsically conducting polymers, thin layers) are related to the reflection of electromagnetic waves (Kim et al. 2002; Saini et al. 2009; Tantawy et al. 2013; Shahzad et al. 2016). Absorption of electromagnetic waves occurs for materials with high dielectric permittivity or high magnetic permeability (Khan et al. 2014). Many materials with high EMI shielding or MA properties contain nanoparticles, which can be dangerous for environment or human health. Development of low cost and eco-friendly materials with high EMI and MA properties is a big challenge. The major requirements for a good microwave absorber are high reflection loss and high absorption of electromagnetic energy. Moreover, good absorber should be characterized by high stability and ability to absorb radiation in broad frequency and temperature range. However, many studies in literature are performed only for room temperature, which is associated with limits of used network analyzers (Cui et al. 2017; Chen et al. 2018; Yang et al. 2018). Results presented in this work suggest that the microwave absorbers are very sensitive to temperature changes.

The aim of this work was to study dielectric properties, electrical conductivity and MA properties of amorphous yellow dextrin in order to show its usefulness in EMI shielding applications. Additionally, the influence of water, temperature and absorber thickness on properties of dextrin-based absorbers was tested and described for microwave frequency region. It is assumed that the addition of water causes changes in relaxation processes in the yellow dextrin, which is manifested by the changes in electrical conductivity and microwave absorption properties. The described observation may be helpful for preparation of new organic matrices with very high dielectric losses and a strictly defined absorption region.

At present, there are good materials that can be used as EMI shielding agents for frequencies higher than $1 \mathrm{GHz}$. The $\mathrm{Fe}_{3} \mathrm{O}_{4}$ nanoparticles are widely used for frequencies above $1 \mathrm{GHz}$, due to their great magnetodielectric properties. However, as there are many technologies operating at lower frequency range i.e. $100 \mathrm{MHz}$ to $1 \mathrm{GHz}$ region, it would be beneficial to deliver appropriate material working in mentioned range. This technological aspect was a major motivation to start studies on polycarbohydrates, compounds which are able to cover the gap in the bottom microwave region due to their extraordinary dielectric properties. For the first study dextrin has been chosen as a representative of polysaccharide group.

\section{Materials and methods}

The fully amorphous dextrin was obtained by ball milling process of yellow dextrin (Venus Starch Suppliers, India) at room temperature. The process was conducted for $8 \mathrm{~h}$ in the Fritsch Pulverisette 6 planetary mill with use of agate bowl and balls. The rotation speed was $450 \mathrm{rpm}$ and process was conducted in cycles of 5 min milling and 5 min pause to avoid overheating of the material. After the milling, the sample was heated up to $373 \mathrm{~K}$ to remove water (sample marked as dextrin-dry). The water adsorption process in environmental chamber was used to obtain second sample marked as dextrin-wet. The process was conducted at $298 \mathrm{~K}$ by $2 \mathrm{~h}$ with $90 \%$ humidity. The amount of water in the sample was determined by thermogravimetric analysis (TGA) performed by 
thermal analyzer NETSCH Jupiter STA 449 F3. The verification of amorphous phase was performed using X-ray diffractometer Rigaku MiniFlex 600 with a copper tube $\mathrm{Cu} \mathrm{K} \alpha(\lambda=0.15406 \mathrm{~nm})$. Novocontrol Concept 81 dielectric spectrometer equipped with Novocontrol Alfa and Keysight 4991E analyzers and Novocool temperature control system was used to determine dielectric, EMI shielding and MA properties of dextrin. Complex dielectric permittivity was measured in broad frequency range: $5 \times 10^{-2}-$ $1 \times 10^{6} \mathrm{~Hz}$ to determine electrical properties and relaxation processes in amorphous yellow dextrin and $5 \times 10^{6}-3 \times 10^{9} \mathrm{~Hz}$ to determine microwave absorption properties. Additionally, microwave absorption properties of dextrin up to $10 \mathrm{GHz}$ were determined based on the extrapolation of measured complex dielectric permittivity. The frequency-temperature superposition was used to determine complex permittivity in the frequency range 3-10 GHz. All the spectra were fitted to the Havriliak-Negami function which was further extrapolated to the desired region. All the measurements were performed in broad temperature range $173-333 \mathrm{~K} \quad(\Delta T=10 \mathrm{~K}$ for $173-203 \mathrm{~K}$ and $\Delta T=5 \mathrm{~K}$ for $203-333 \mathrm{~K}$ ).

\section{Results and discussion}

The amorphous structure of yellow dextrin was verified using XRD method (see Fig. 1a for details). The starting material was partially amorphous, however after ball milling, only single amorphous halo can be observed both for dextrin-dry and dextrin-wet samples. On the basis of thermogravimetric (TGA) curves (Fig. 1b) amount of water in samples was calculated from the mass loss curves and was equaled to $4 \%$ for starting material, $0.4 \%$ for dextrin-dry and $5.4 \%$ for dextrin-wet.

To determine influence of water and temperature on the electrical properties as well as on relaxation processes, real part $\left(\varepsilon^{\prime}\right)$ and imaginary part $\left(\varepsilon^{\prime \prime}\right)$ of dielectric permittivity were measured and presented in Fig. 2a-d. It can be noticed, that the presence of water in sample significantly increases values of $\varepsilon^{\prime}$ and $\varepsilon^{\prime \prime}$ in the low frequency range. This phenomenon is associated with two processes: increased electrical conductivity and electrode polarization. It can be clearly seen in Fig. 2e, f, in which real part of AC electrical conductivity $\left(\sigma_{A C}\right)$ was presented. It can be noticed,
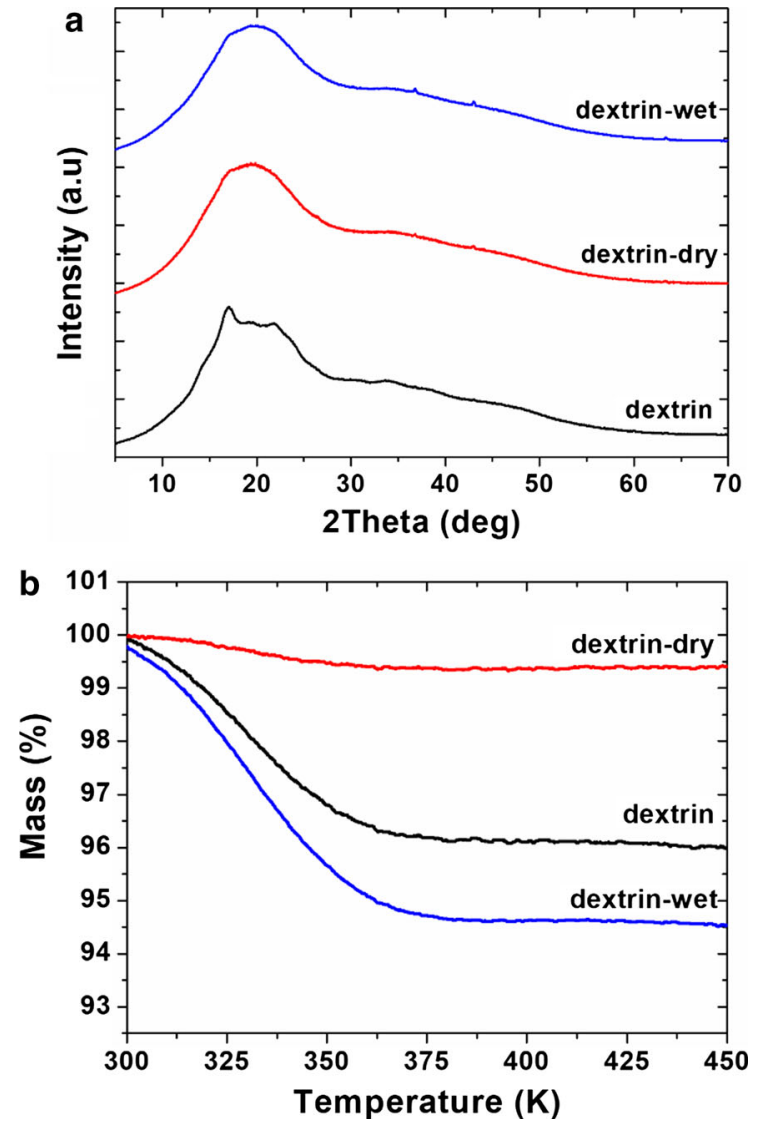

Fig. 1 a XRD patterns for yellow dextrin, milled and dried dextrin (dextrin-dry) and dextrin-dry saturated with water (dextrin-wet); b TGA curves for all samples

that for dextrin-dry and dextrin-wet two different regions can be observed at elevated temperature. The first one is plateau region which is observed at low frequencies and is associated with DC conductivity of sample (up to $10 \mathrm{~Hz}$ at $T=333 \mathrm{~K}$ ). The second one, observed at high frequencies is the dispersion of $\mathrm{AC}$ electrical conductivity (for $f>10 \mathrm{~Hz}$ at $T=333 \mathrm{~K}$ ). Additionally, plateau region can be observed even for lower temperatures for dextrin-wet, which is related to the presence of water in sample. Basing on the TG analysis it can be concluded, that this enormous increase of conductivity for dextrin-wet is related to the increased number of mobile water molecules. Presence of water enhances proton hopping-the process that most probably is responsible for the conductivity in the analyzed samples. The changes in conduction mechanism of yellow dextrin can be clearly visible in plots of imaginary part of electric 
Fig. 2 Dielectric properties of dextrin-dry and dextrinwet: real part $(\mathbf{a}, \mathbf{b})$ and imaginary part $(\mathbf{c}, \mathbf{d})$ of permittivity; AC electrical conductivity $(\mathbf{e}, \mathbf{f})$ and imaginary part of electric modulus $(\mathbf{g}, \mathbf{h})$
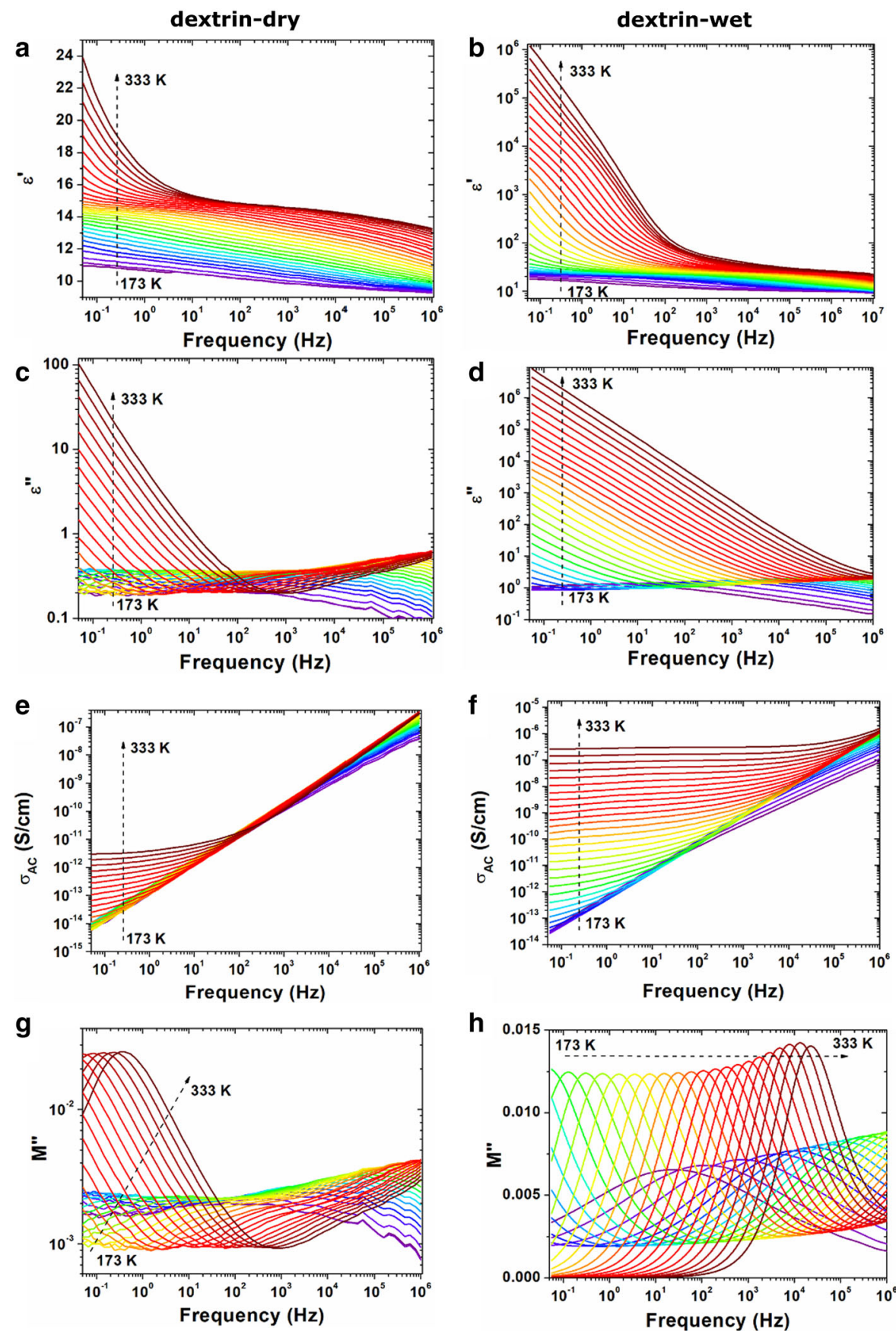

modulus $\left(M^{\prime \prime}\right)$ for wet and dry dextrin (Fig. $2 \mathrm{~g}, \mathrm{~h}$ ). Modulus peak is associated with the conduction relaxation processes and can be expressed by the Eq. (1) (McCrum et al. 1968; Macedo et al. 1972; Howell et al. 1974).

$$
M^{\prime \prime}=\frac{\varepsilon^{\prime \prime}}{\left(\varepsilon^{\prime}\right)^{2}+\left(\varepsilon^{\prime \prime}\right)^{2}}
$$

Characteristic relaxation time $\left(\tau_{M^{\prime \prime}}\right)$ of conductivity peaks was calculated applying formula: $\tau_{M^{\prime \prime}}=1 / 2 \pi f$ and presented in function of temperature for dextrin- 

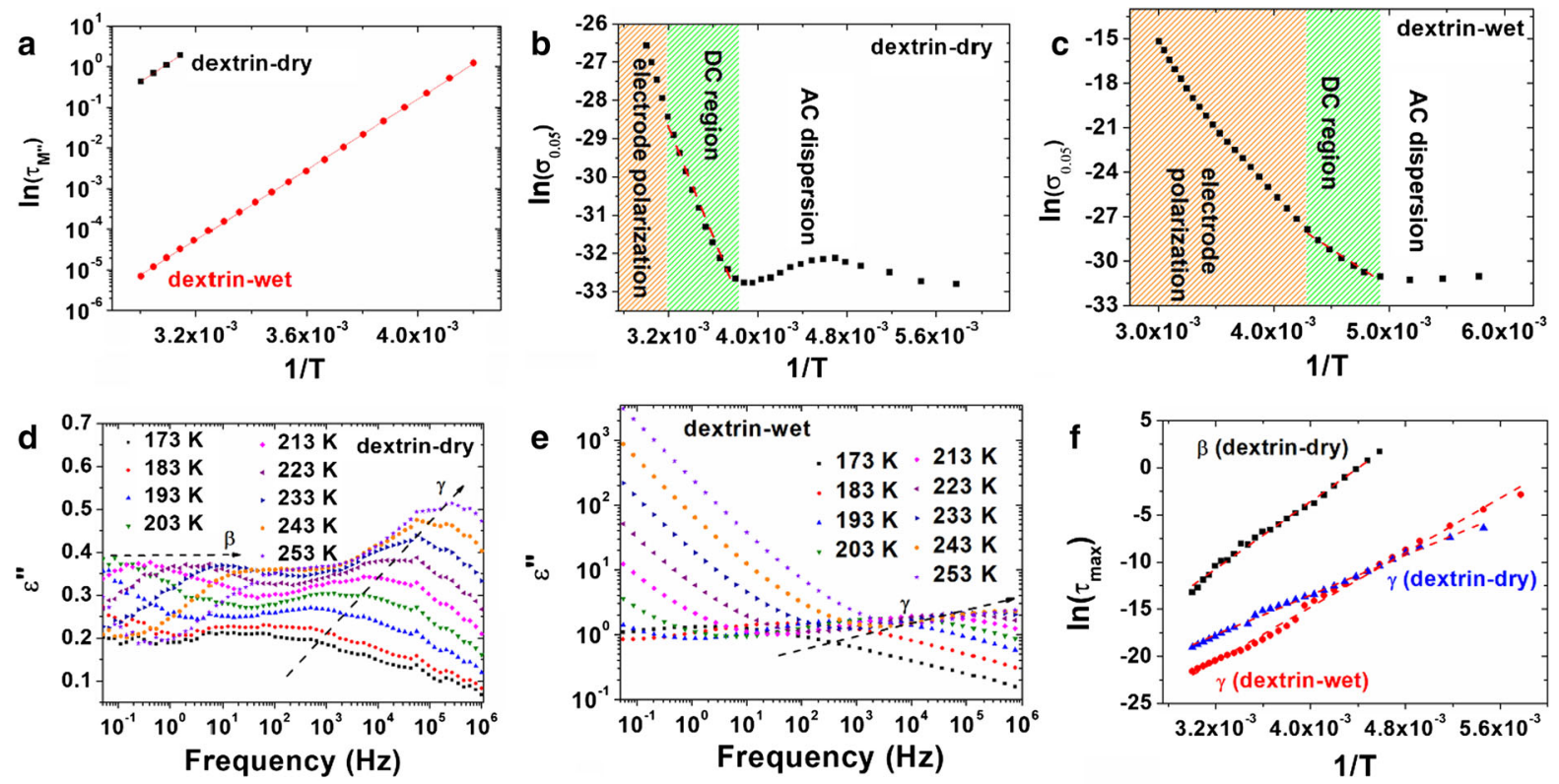

Fig. 3 a Arrhenius plots of relaxation time corresponding to the transition between electrode polarization process and DC electrical conductivity; b, c Arrhenius plots (red dashed lines) of DC electrical conductivity associated with proton transfer process for dextrin-dry (b) and dextrin-wet (c); d, e plots of

wet and dextrin-dry in Fig. 3a. It can be noticed, that for dextrin-dry the peak of $M^{\prime \prime}$ can be observed only for higher temperatures (above $318 \mathrm{~K}$ ), whereas for dextrin-wet even at $238 \mathrm{~K}$. It can be noticed, that the Arrhenius law can be used to determine activation energy $(\Delta E)$ of this process (Eq. $2 ; k_{B}$ is the Boltzmann constant, $T$ is the temperature and $\tau_{0}$ is the preexponential factor). For dextrin-dry the activation energy of this process is slightly higher, which can be associated with low amount of water in the sample (Table 1).

Table 1 Activation energies of different processes: transition between electrode polarization and DC electrical conductivity $(\mathrm{EP}-\mathrm{DC})$, proton transfer $(\mathrm{H}), \beta$-relaxation $(\beta)$ and $\gamma$-relaxation $(\gamma)$

\begin{tabular}{lll}
\hline Process & $\Delta \mathrm{E}$ (dextrin-dry) $(\mathrm{eV})$ & $\Delta \mathrm{E}$ (dextrin-wet) $(\mathrm{eV})$ \\
\hline EP-DC & 0.39 & 0.37 \\
$\mathrm{H}$ & 0.62 & 0.44 \\
$\beta$ & 0.77 & Not observed \\
$\gamma$ & 0.45 & 0.62 \\
\hline
\end{tabular}

imaginary part of permittivity at low temperature region (173-253 K) for dextrin-dry (d) and dextrin-wet (e) with marked $\beta$ and $\gamma$-relaxation processes; $\mathbf{f}$ Arrhenius plots of relaxation time corresponding to the $\beta$ and $\gamma$-relaxation processes. (Color figure online)

$\tau=\tau_{0} \times \exp \left(\frac{\Delta E}{k_{B} T}\right)$

Taking into account the presence of electrode polarization and DC conductivity $\left(\sigma_{D C}\right)$ contribution, it is also possible to determine the energy of the proton transfer-a process related to the $\sigma_{D C}$. For this purpose slope of the Arrhenius plot $\left[\ln \left(\sigma_{0.05}\right)\right.$ vs. $1 / T$, where $\sigma_{0.05}$ is the AC electrical conductivity at $0.05 \mathrm{~Hz}$ ], for region in which electrode polarization doesn't occur was determined. Results have been presented in Fig. 3b, c (Isa and Ahmad 2018). The much higher activation energy of this process $\left(\Delta E_{D C}\right)$ for dextrindry $(0.62 \mathrm{eV})$ is associated with very low amount of water molecules in sample ( $0.4 \mathrm{wt} \%$ according to the TGA curve). As it was expected, the presence of water lowers the proton hopping barrier (activation energy equaled to $0.44 \mathrm{eV}$ ), so electrical conductivity is much higher and electrode polarization occurs at higher temperatures (Nguyen et al. 2016).

Two different relaxation processes can be also observed in the $\varepsilon^{\prime \prime}(f)$ plots for lower temperature region (Fig. 3d, e). These processes are associated 
with $\beta$ and $\gamma$-relaxations in yellow dextrin. One can observe their shift to higher frequencies with increasing temperature. The water causes suppression of $\beta$ relaxation and improves dielectric strength of $\gamma$ relaxation. This behavior is universal for the saccharide family (Einfeldt and Kwasniewski 2002; Kaminski et al. 2012). As it was earlier stated in the introduction section, $\beta$-relaxation is probably related to the monosaccharide unit rotations via the glycosidic linkage, while $\gamma$-relaxation can be correlated with the orientational changes of hydroxymethyl substituents. The water is a factor that alters hydrogen bonding patterns. Sugar-water hydrogen bonds are stronger than bonds between sugar-sugar and in consequence, sugar internal hydrogen bonds are significantly suppressed. Lower stiffness of glycosidic bridge in wet sugar is related to the lower activation energy for $\beta$ relaxation. In case of yellow dextrin this relaxation is not observed when sample is saturated with water. As it is much faster and it's dielectric strength is lower it can be covered by strong $\gamma$-relaxation which is boosted by the presence of water. The water bound to $-\mathrm{CH}_{2} \mathrm{OH}$ groups by hydrogen bonds causes enhancement of energy barrier for conformational interconversion of these groups. The activation energies of these processes were calculated according to the Eq. (2) with relaxation times determined using Havriliak-Negami function (Eq. 3) and presented in the Fig. 3f.

$\varepsilon^{*}(\omega)=\varepsilon+\frac{\Delta \varepsilon}{\left[1+\left(i \omega \tau_{H N}\right)^{\alpha}\right]^{\beta}}$

where $\varepsilon^{*}$ is the complex permittivity, $\alpha$ and $\beta$ are the shape parameters, $\Delta \varepsilon$ is the dielectric strength and $\tau_{H N}$ is the Havriliak-Negami relaxation time. Relaxation time $\tau_{\max }$ of analyzed process is given by the equation:

$\tau_{\max }=\tau_{H N}\left[\frac{\sin \left(\frac{\pi \alpha \beta}{2(\beta+1)}\right)}{\sin \left(\frac{\pi \alpha}{2(\beta+1)}\right)}\right]^{\frac{1}{\alpha}}$

It can be noticed, that at room temperature $\gamma$ relaxation in wet sample is faster (shifted to higher frequencies). The calculated $\Delta E$ of these processes are presented in Table 1 . In case of dextrin-wet $\gamma$-relaxation has much higher dielectric strength $(\Delta \varepsilon)$ when compared to dried sample. At $298 \mathrm{~K}$ dielectric strength is equals 3.79 for dry dextrin and 16.36 for wet.

High frequency dielectric properties of dextrin-dry and dextrin-wet were presented in Fig. 4. It can be noticed, that with increasing temperature the values of real part and imaginary part of permittivity increase. Additionally, $\gamma$-relaxation in higher frequencies can be observed and presence of water increases significantly values of both $\varepsilon^{\prime}$ and $\varepsilon^{\prime \prime}$. These changes should have significant impact on the microwave absorption properties. Therefore, EMI shielding and MA properties were determined from the measurements of complex permittivity for dry and wet material. For this purpose, two key factors were calculated: reflection loss $(R L)$, which is associated with absorption properties of material and expresses the ratio of reflected power to incident power and EMI shielding effectiveness $(S E)$, which is defined as the ratio of transmitted power to incident power (Zhang et al. 2012).

For one layer absorber backed by a copper plate, the $R L$ is the most important factor, which determines the MA properties of absorber and it depends on complex electric permittivity, complex magnetic permeability, layer thickness and temperature. Generally, $R L$ and $S E$ for a single layer absorber backed by a copper plate can be expressed as (Qin and Brosseau 2012; Zhang et al. 2012):

$$
\begin{aligned}
& R L=20 \log |\Gamma| \\
& R L(\%)=100-100\left(10^{\frac{R L}{20}}\right)^{2} \\
& S E=-20 \log \left|\frac{\left(1-\Gamma^{2}\right) T}{1-T^{2} \Gamma^{2}}\right| \\
& \Gamma=\frac{\sqrt{\frac{\left(\mu^{\prime}-j \mu^{\prime \prime}\right)}{\left(\varepsilon^{\prime}-j \varepsilon^{\prime \prime}\right)}}}{\sqrt{\frac{\left(\mu^{\prime}-j \mu^{\prime \prime}\right)}{\left(\varepsilon^{\prime}-j \varepsilon^{\prime \prime}\right)}} \tanh \left(j \frac{2 \pi f d}{c} \sqrt{\left(\mu^{\prime}-j \mu^{\prime \prime}\right)\left(\varepsilon^{\prime}-j \varepsilon^{\prime \prime}\right)}\right)-1}
\end{aligned}
$$

$T=e^{-\left(j \frac{2 \pi f d}{c} \sqrt{\left(\mu^{\prime}-j \mu^{\prime \prime}\right)\left(\varepsilon^{\prime}-j \varepsilon^{\prime \prime}\right)}\right)}$

where $\Gamma$ is the reflection coefficient, $T$ is the transmission coefficient, $\varepsilon^{\prime}$ and $\varepsilon^{\prime \prime}$ are the real and imaginary parts of complex electric permittivity, $\mu^{\prime}$ and $\mu^{\prime \prime}$ are the real and imaginary parts of complex magnetic permeability, $f$ is the frequency of electromagnetic radiation, $c$ is the speed of light and $d$ is the absorber thickness. For diamagnetic materials, in which MA is due to dielectric losses, complex permeability can be expressed as $\mu^{\prime}=1-j 0$. 
Fig. 4 Plots of real part (a, c) and imaginary part (b, d) of permittivity in broad temperature region $(173 \mathrm{~K}-$ $333 \mathrm{k})$ for dextrin dry (a, b) and dextrin-wet $(\mathbf{c}, \mathbf{d})$
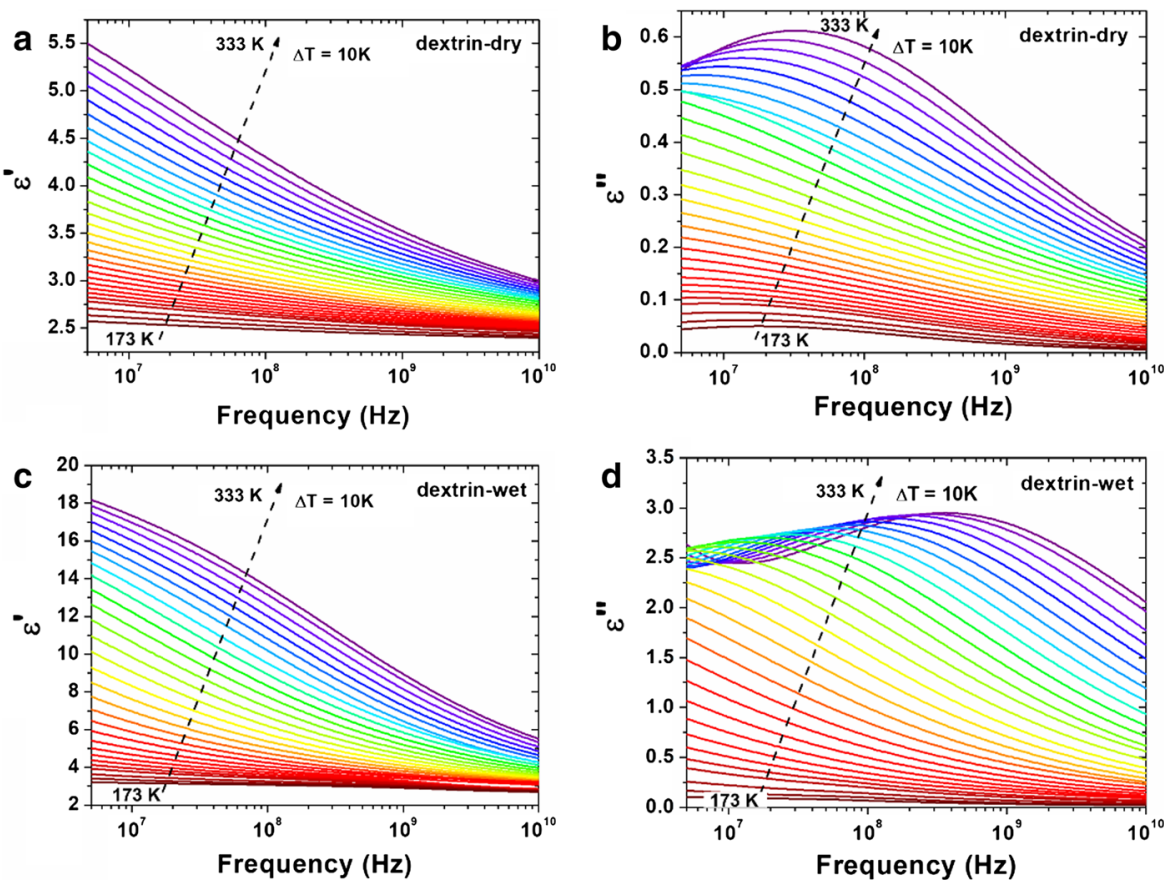

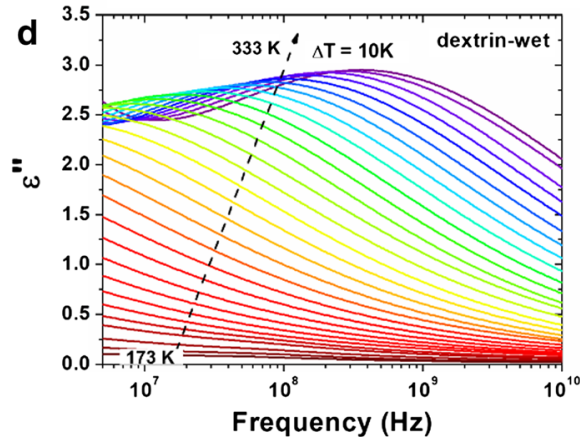

The results of analysis were presented in Fig. 5. Figure 5a, b shows $R L$ for dry and wet yellow dextrin calculated for different values of absorber thickness at constant temperature ( $298 \mathrm{~K}$ ). It can be noticed, that with increasing $d$, values of $S E$ and $R L$ decrease. The more negative the value of these parameters, the greater the absorption properties. However, absorption extremum can be observed for different absorber thicknesses at different frequencies (Table 2). Moreover, the most negative value of $R L$ for dextrin-dry was observed only for absorber with thickness $8-10 \mathrm{~cm}$, but obtained $R L$ was much lower than $R L_{\text {min }}$ for dextrin-wet (Table 2). The most negative value of $S E$ was observed for layer thickness near $10 \mathrm{~cm}$, but $S E$ lower than $-20 \mathrm{~dB}$ was observed also for lower value of $d$ (Fig. 5c, d). However, as can be seen in Fig. 5 dextrin-wet is characterized by very negative $R L$ and $S E$ even for low frequencies and absorber thickness.

Temperature dependence of the $R L$ and $S E$ was also determined for dextrin-dry and dextrin-wet (Fig. 6). Additionally, skin depth, defining how deep is the penetration of material by the electromagnetic radiation, was calculated in wide frequency region i.e. $5 \mathrm{MHz}-10 \mathrm{GHz}$. This factor is defined as the depth at which the intensity of the radiation inside the material falls to $1 / e$ of its original value at the surface. For diamagnetic materials it can be expressed as:

$\delta=\frac{c \sqrt{\varepsilon^{\prime}}}{2 \pi f \varepsilon^{\prime \prime}}$

It can be noticed, that the water presented in sample decreases the value of $\delta$ (especially in the gigahertz region). Additionally, $\delta$ decreases also with increasing temperature. Dextrin-wet has much lower value of $\delta$ and in consequence much thinner absorbers can be produced from it when compared to the dextrin dry.

Microwave absorption properties of dielectric materials are associated with processes induced by an electromagnetic radiation. These processes allow to dissipate electromagnetic radiation by converting it into thermal energy and are frequency and temperature dependent (Zhao et al. 2013). It can be easily noticed that $R L$ and $S E$ parameters, which were presented in Fig. $6 \mathrm{c}-\mathrm{f}$ are sensitive to temperature change. For example, absorption extremum $(-17.29 \mathrm{~dB})$ observed for dextrin-wet at $333 \mathrm{~K}$ increases to $-5.47 \mathrm{~dB}$ at $298 \mathrm{~K}$ and to $-0.04 \mathrm{~dB}$ at $173 \mathrm{~K}$ (Fig. 6d). The same tendency was observed for dextrin-dry, for which absorption extremum increases from $-2.37 \mathrm{~dB}$ at $333 \mathrm{~K}$ to $-0.06 \mathrm{~dB}$ at $173 \mathrm{~K}$ (Fig. 6c). It was noticed, that a water presented in sample (at only $5.4 \mathrm{wt} \%$ ) significantly increases 

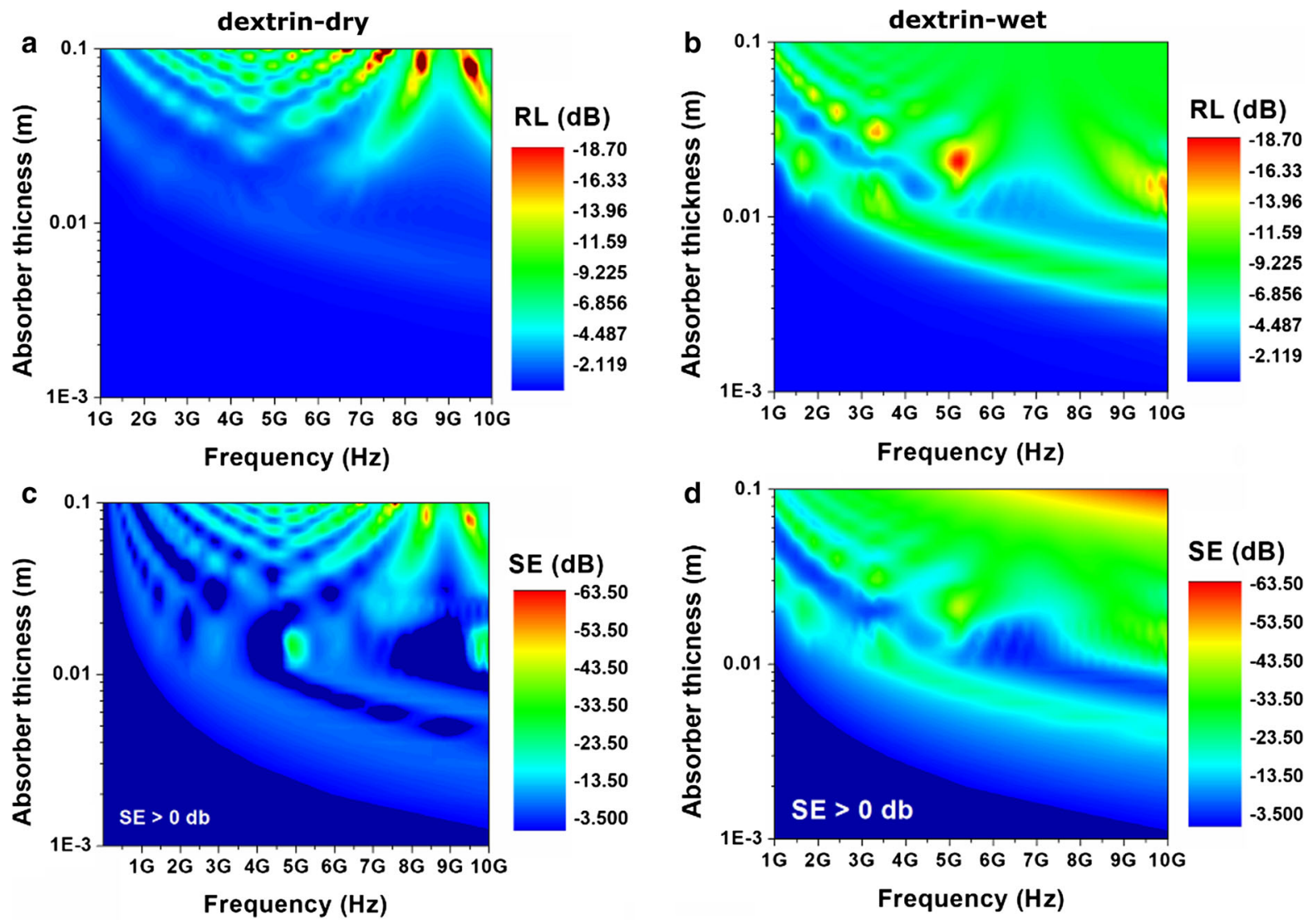

Fig. 5 The reflection loss $R L$ and shielding effectiveness $S E$ calculated for dry $(\mathbf{a}, \mathbf{c})$ and wet (b, d) yellow dextrin with different thickness $(1 \mathrm{~mm}-10 \mathrm{~cm})$ at constant $\mathrm{T}=298 \mathrm{~K}$

Table 2 The values of $R L_{\min }$ calculated for wet and dry yellow dextrin with corresponding frequencies and absorber thickness d

\begin{tabular}{llccc}
\hline Sample & Frequency $(\mathrm{GHz})$ & $d(\mathrm{~mm})$ & $R L_{\min }(\mathrm{dB})$ & $R L(\%)$ \\
\hline Dextrin-wet & 1.89 & 50 & -14.43 & 96.40 \\
& 2.42 & 40 & -15.21 & 96.89 \\
& 3.30 & 30 & -16.30 & 97.60 \\
Dextrin-dry & 5.24 & 20 & -18.58 & 98.60 \\
& 6.66 & 100 & -29.43 & 99.89 \\
& 7.56 & 100 & -35.03 & 99.97 \\
& 8.37 & 80 & -24.22 & 99.62 \\
& 9.50 & 80 & -28.56 & 99.86 \\
\hline
\end{tabular}

microwave absorption properties of dextrin. The value of $R L_{\text {min }}$ increases from -17.29 to $-2.37 \mathrm{~dB}$ and $S E_{\text {max }}$ from -36.84 to $-9.09 \mathrm{~dB}$ when comparing samples with and without water. Additionally, water causes the shift of absorption extremum from $4.78 \mathrm{GHz}$ in dry dextrin to $2.83 \mathrm{GHz}$ in wet dextrin. Moreover, from the frequency dependent $R L$ diagram presented in Fig. $7 \mathrm{a}$, b one can note that $R L_{\text {min }}$ shifts to lower frequencies with increasing temperature. For the wet dextrin at elevated temperature (above $323 \mathrm{~K}$ ) two absorption maxima can be observed: at $2.83 \mathrm{GHz}$ and at $9.12 \mathrm{GHz}$ (for $333 \mathrm{~K}$ ). 

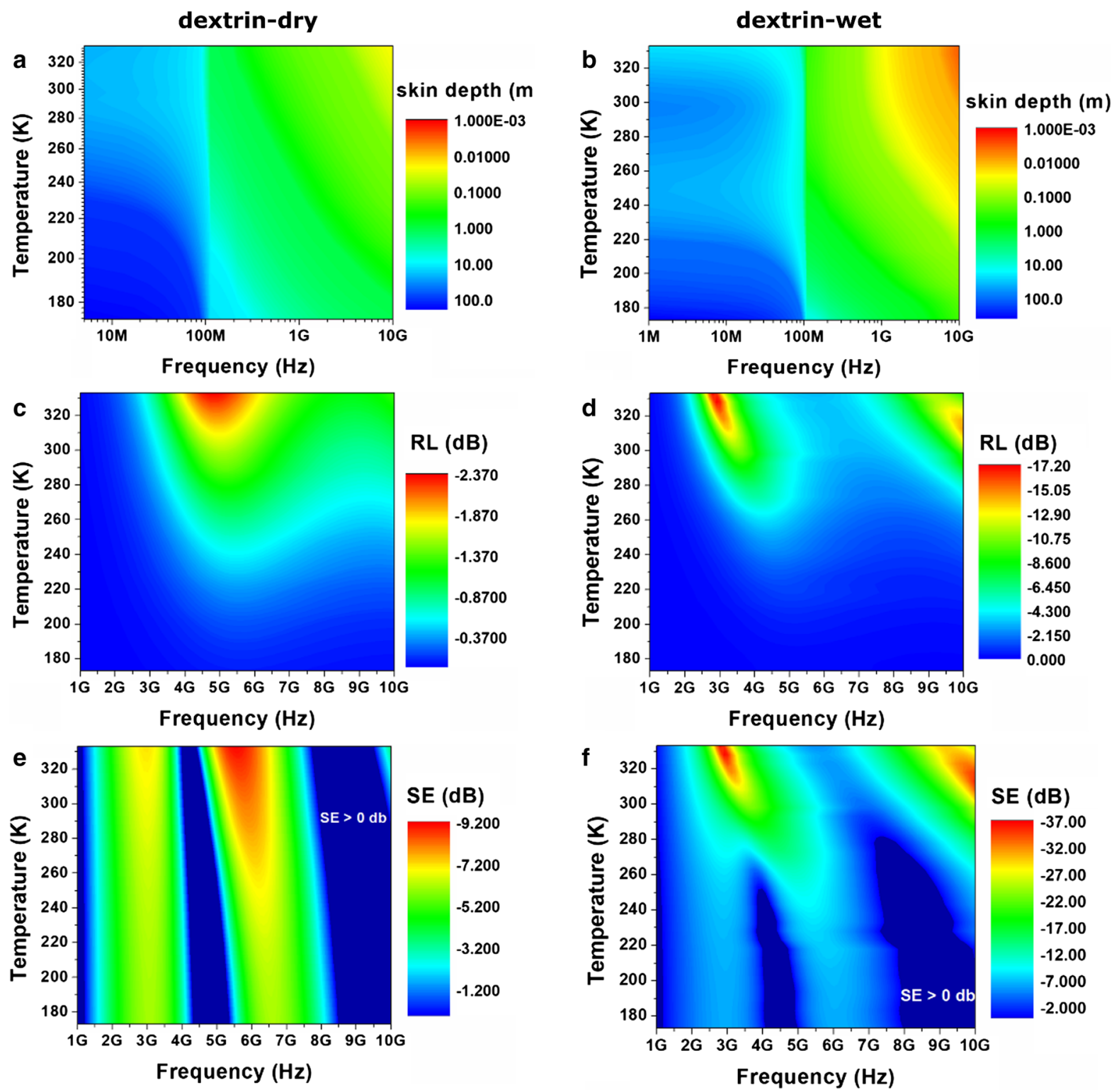

Fig. 6 The temperature dependent skin depth, reflection loss and shielding effectiveness calculated for dry (a, c, e) and wet (b, d, f) yellow dextrin with $d=1 \mathrm{~cm}$

\section{Conclusions}

In this work, dielectric properties, electrical conductivity and microwave absorption properties of amorphous yellow dextrin were described. The influence of water and temperature on these parameters was described in detail. It was noticed, that a minor addition of water significantly improves MA properties, which were related to the shift of $\gamma$-relaxation to the higher frequencies for dextrin containing water. It was noticed, that the shift of this process improves significantly microwave absorption properties of amorphous yellow dextrin. The value of $R L_{\text {min }}$ decreases from -2.37 up to $-17.29 \mathrm{~dB}$ and $S E_{\max }$ from -9.09 up to $-36.84 \mathrm{~dB}$ for sample containing only $5.4 \mathrm{wt} \%$ of water. This observation may be used for preparation of new organic matrices with very high dielectric losses and a strictly defined absorption region. Additionally, it was confirmed that the microwave absorption properties are temperature 

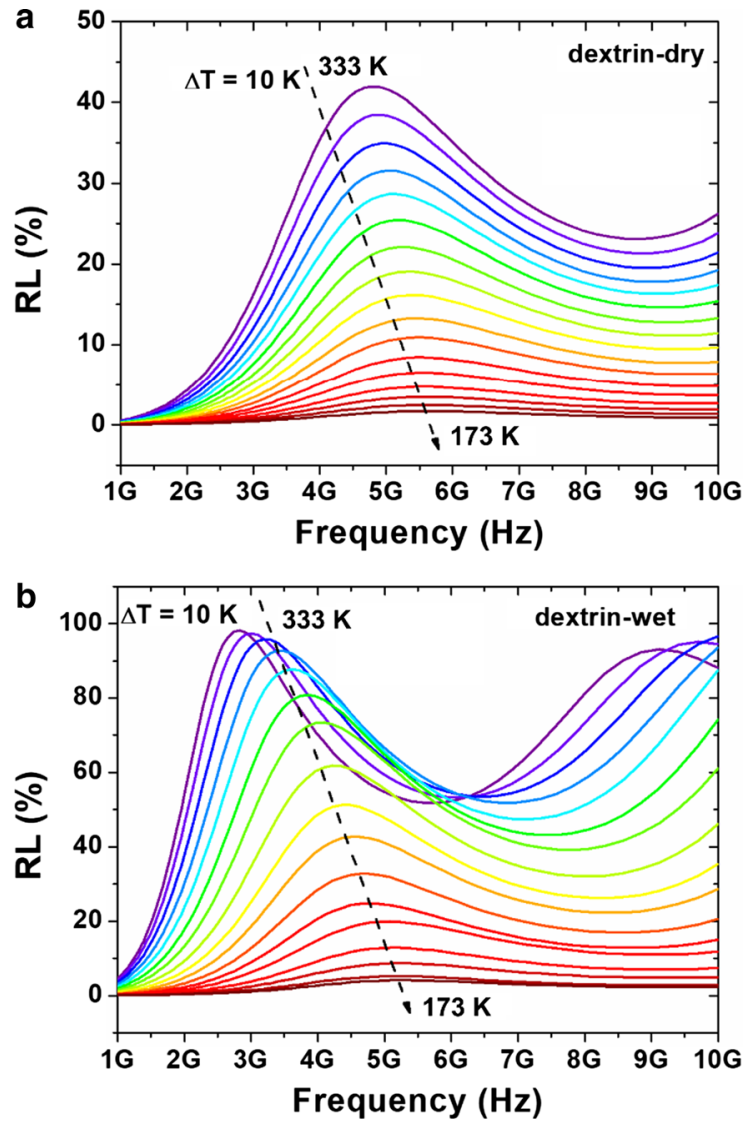

Fig. 7 The percentage reflection loss calculated for wet (a) and dry (b) yellow dextrin at wide temperature range 173-333 K

dependent. It was noticed, that $R L_{\min }$ shifts to lower frequencies with increasing temperature. Moreover, it was clearly confirmed, that the motion of water molecules in sample improves DC electrical conductivity and is responsible for electrode polarization process. According to the analysis of imaginary part of electric modulus, relaxation time of transition between electrode polarization and DC electrical conductivity was determined and by the use of Arrhenius plots, energy of activation of this process was calculated. For dextrin-dry the activation energy of this process is slightly higher $(0.39$ and $0.37 \mathrm{eV}$ for dextrin-dry and dextrin-wet, respectively), which can be associated with low amount of water in sample (up to $0.4 \%$ ). Moreover, activation energy of $\beta$ and $\gamma$-relaxation processes were calculated. The activation energy of $\gamma-$ relaxation increased significantly for sample with water. It was noted, that the $\beta$-relaxation disappears in dextrin-wet. Considering the relationship between AC electrical conductivity and frequency, three regions were observed in $\sigma_{A C}(f)$ plots: (1) associated with electrode polarization, (2) related to the DC electrical conductivity and (3) associated with dispersion of AC electrical conductivity in high frequency region. According to that, the influence of water on the activation energy of proton transfer (the process that most probably is responsible for the DC electrical conductivity in the analyzed samples) was determined for DC region. It was noticed, that a dextrin-wet had much lower $\Delta E_{D C}$ than dextrin-dry $(0.44$ and $0.62 \mathrm{eV}$, respectively), which is related to the presence of mobile water molecules.

The approach proposed in this article can be used in the future to analyze other organic compounds and determine not only the influence of water as well as the ion motions on the electrical conductivity and the occurrence of electrode polarization processes. Moreover, further studies associated with determination of water on the microwave absorption properties of different compounds should lead to the production of an absorber with industrial use.

Open Access This article is distributed under the terms of the Creative Commons Attribution 4.0 International License (http:// creativecommons.org/licenses/by/4.0/), which permits unrestricted use, distribution, and reproduction in any medium, provided you give appropriate credit to the original author(s) and the source, provide a link to the Creative Commons license, and indicate if changes were made.

Funding This work was supported by the National Science Center, Poland [Grant No. 2016/23/B/ST8/03405].

\section{References}

Boruch M, Brzeziński S, Palka A, Pierzgalski T (1988) Measurement of dielectric properties for moisture determination of dextrins. Starch/Starke 10:392-395. https://doi.org/ 10.1002/star.19880401007

Chen C, Pan L, Jiang S et al (2018) Electrical conductivity, dielectric and microwave absorption properties of graphene nanosheets/magnesia composites. J Eur Ceram Soc 38:1639-1646. https://doi.org/10.1016/j.jeurceramsoc. 2017.11.052

Cui R, Pan L, Zhang D, Nasir H (2017) Electromagnetic microwave absorption properties of carbon nanocoils/tissue. Diam Relat Mater 77:53-56. https://doi.org/10.1016/j. diamond.2017.05.014

Einfeldt J, Kwasniewski A (2002) Characterization of different types of cellulose by dielectric spectroscopy. Cellulose 9:225-238. https://doi.org/10.1023/A:1021184620045 
Greenwood CT (1967) The thermal degradation of starch. Adv Carbohydr Chem. https://doi.org/10.1016/s00965332(08)60157-5

Howell FS, Bose RA, Macedo PB, Moynihan CT (1974) Electrical relaxation in a glass-forming molten salt. J Phys Chem. https://doi.org/10.1021/j100599a016

Isa MIN, Ahmad NH (2018) Carboxymethyl cellulose-ammonium chloride on electrical of plasticized propylene carbonate solid bio-polymer electrolytes (SBPs) using experimental and computational studies. Phosphorus Sulfur Silicon Relat Elem 193:115-119. https://doi.org/10. 1080/10426507.2017.1417308

Kaminski K, Kaminska E, Hensel-Bielowka S et al (2008) Identification of the molecular motions responsible for the slower secondary $(\beta)$ relaxation in sucrose. J Phys Chem B. https://doi.org/10.1021/jp711502a

Kaminski K, Wlodarczyk P, Adrjanowicz K et al (2010) Origin of the commonly observed secondary relaxation process in saccharides. J Phys Chem B 114:11272-11281. https://doi. org/10.1021/jp1034773

Kaminski K, Adrjanowicz K, Kaminska E et al (2012) Impact of water on molecular dynamics of amorphous $\alpha-, \beta-$, and $\gamma$ cyclodextrins studied by dielectric spectroscopy. Phys Rev E Stat Nonlinear Soft Matter Phys. https://doi.org/10.1103/ physreve.86.031506

Khan SUD, Arora M, Wahab MA, Saini P (2014) Permittivity and electromagnetic interference shielding investigations of activated charcoal loaded acrylic coating compositions. J Polym 2014:1-7. https://doi.org/10.1155/2014/193058

Kim MS, Kim HK, Byun SW et al (2002) PET fabric/polypyrrole composite with high electrical conductivity for EMI shielding. Synth Met 126:233-239. https://doi.org/10. 1016/S0379-6779(01)00562-8

Macedo PB, Moynihan CT, Bose R (1972) Role of ionic diffusion in polarization in vitreous ionic conductors. Phys Chem Glas. https://doi.org/10.4236/ns.2014.66038

McCrum N, Williams B, Read G (1968) Anelastic and dielectric effects in polymeric solids. Polymer (Guildf). https://doi. org/10.1016/0032-3861(68)90070-0

Nguyen HT, Sidorkin AS, Milovidova SD, Rogazinskaya OV (2016) Influence of humidity on dielectric properties of nanocrystalline cellulose-triglycine sulfate composites. Ferroelectrics 501:180-186. https://doi.org/10.1080/ 00150193.2016 .1204866

Qin F, Brosseau C (2012) A review and analysis of microwave absorption in polymer composites filled with carbonaceous particles. J Appl Phys 111:4

Rachocki A, Markiewicz E, Tritt-Goc J (2005) Dielectric relaxation in cellulose and its derivatives. Acta Phys Pol A 108:137-145

Saini P, Choudhary V, Singh BP et al (2009) PolyanilineMWCNT nanocomposites for microwave absorption and EMI shielding. Mater Chem Phys 113:919-926. https://doi. org/10.1016/j.matchemphys.2008.08.065

Shahzad F, Alhabeb M, Hatter CB et al (2016) Electromagnetic interference shielding with $2 \mathrm{D}$ transition metal carbides
(MXenes). Science 353:1137-1140. https://doi.org/10. 1126/science.aag2421

Shinouda HG, Moteleb MMA (2005) Dielectric spectroscopy and relaxation phenomena of moistened and dry polysaccharides. J Appl Polym Sci 98:571-582. https://doi.org/10. 1002/app.22021

Shrestha AK, Halley PJ (2014) Starch modification to develop novel starch-biopolymer blends: state of art and perspectives. In: Halley PJ, Avérous L (eds) Starch polymers: from genetic engineering to green applications. Elsevier, UK, pp 105-143. https://doi.org/10.1016/B978-0-444-53730-0. 00022-1

Silva DM, Nunes C, Pereira I et al (2014) Structural analysis of dextrins and characterization of dextrin-based biomedical hydrogels. Carbohydr Polym. https://doi.org/10.1016/j. carbpol.2014.08.009

Takatori Y, Akagi S, Sugiyama H et al (2011) Icodextrin increases technique survival rate in peritoneal dialysis patients with diabetic nephropathy by improving body fluid management: a randomized controlled trial. Clin J Am Soc Nephrol. https://doi.org/10.2215/cjn.10041110

Tantawy HR, Aston DE, Smith JR, Young JL (2013) Comparison of electromagnetic shielding with polyaniline nanopowders produced in solvent-limited conditions. ACS Appl Mater Interfaces 5:4648-4658. https://doi.org/10. 1021/am401695p

Terpstra KR, Woortman AJJ, Hopman JCP (2010) Yellow dextrins: evaluating changes in structure and colour during processing. Starch/Starke. https://doi.org/10.1002/star. 200900254

Weingärtner H, Knocks A, Boresch S et al (2001) Dielectric spectroscopy in aqueous solutions of oligosaccharides: experiment meets simulation. J Chem Phys 115:1463-1472. https://doi.org/10.1063/1.1380205

Wlodarczyk P, Hawelek L, Paluch M et al (2015) Dielectric properties of glassy disaccharides for electromagnetic interference shielding application. J Appl Phys. https://doi. org/10.1063/1.4935271

Yang N, Zeng J, Xue J et al (2018) Strong absorption and widefrequency microwave absorption properties of the nanostructure zinc oxide/zinc/carbon fiber multilayer composites. J Alloys Compd 735:2212-2218. https://doi.org/10. 1016/j.jallcom.2017.11.380

Zhang W, Xu Y, Yuan L et al (2012) Microwave absorption and shielding property of composites with FeSiAl and carbonous materials as filler. J Mater Sci Technol 28:913-919. https://doi.org/10.1016/S1005-0302(12)60150-9

Zhao X, Zhang Z, Wang L et al (2013) Excellent microwave absorption property of graphene-coated $\mathrm{Fe}$ nanocomposites. Sci Rep 3:3421. https://doi.org/10.1038/srep03421

Publisher's Note Springer Nature remains neutral with regard to jurisdictional claims in published maps and institutional affiliations. 